\title{
Warm Molecular Hydrogen and Ionized Neon in the HH 2 Outflow ${ }^{1}$
}

\author{
B. Lefloch ${ }^{1}$ \\ Laboratoire d'Astrophysique, Observatoire de Grenoble, BP 53, F-38041 Grenoble Cédex 9, \\ France \\ Bertrand.Lefloch@obs.ujf-grenoble.fr \\ J. Cernicharo ${ }^{2}$ \\ Consejo Superior de Investigaciones Científicas, Instituto de Estructura de la Materia, \\ Serrano 123, E-28006 Madrid \\ S. Cabrit ${ }^{3}$ \\ LERMA, Observatoire de Paris, UMR 8112 du CNRS, France3 \\ A. Noriega-Crespo ${ }^{4}$ \\ SIRTF Science Center, Caltech JPL, Pasadena, CA 91125, USA \\ A. Moro-Martín ${ }^{5}$ \\ Steward Observatory, University of Arizona, Tucson, AZ, 85721, USA \\ D. Cesarsky $^{6}$ \\ Max-Planck Institut für Extraterrestrische Physik, 85741 Garching, Germany
}

\begin{abstract}
We report on spectro-imaging observations of the Herbig-Haro 2 outflow with the ISOCAM camera onboard the Infrared Space Observatory (ISO). The [Ne II] $12.81 \mu \mathrm{m}$ and [Ne III] $15.55 \mu \mathrm{m}$ lines are detected only towards the jet working surface $(\mathrm{HH} 2 \mathrm{H})$, consistent with the high excitation of this knot in the optical range, while $\mathrm{H}_{2}$ pure rotational emission is found all over the shocked region $\mathrm{HH} 2$. The low energy transition $\mathrm{S}(2)$ traces warm gas $(\mathrm{T} \sim 400 \mathbf{K})$ peaked towards knots E-F and extended ejecta $(\mathrm{T} \sim 250-380 \mathrm{~K})$ with masses of a few $10^{-3} M_{\odot}$ in the high-velocity $\mathrm{CO}$ outflow extending between the powering source and $\mathrm{HH}$ 2. Such emission could arise from low-velocity C-type shocks
\end{abstract}


$\left(\mathrm{v} \simeq 10-15 \mathrm{~km} \mathrm{~s}^{-1}\right)$. The higher transitions $\mathrm{S}(3)$-S $(7)$ trace the emission of hot shocked gas $(\mathrm{T}=1000-1400 \mathrm{~K})$ from individual optical knots in the HH 2 region. The ortho to para (OTP) ratio exhibits large spatial variations between $1.2(\mathrm{E})$ and $2.5(\mathrm{H})$, well below its value at LTE. The emission of the $\mathrm{S}(3)$ $\mathrm{S}(7)$ lines is well accounted for by planar C-shock models with a typical velocity $\mathrm{V}_{\mathrm{s}}=20-30 \mathrm{~km} \mathrm{~s}^{-1}$ propagating into a medium of density $\mathrm{n}_{\mathrm{i}}=10^{4}-10^{5} \mathrm{~cm}^{-3}$ with an initial OTP ratio close to 1 in the pre-shock gas. In the leading edge of the jet, where the geometry of the emission allows a simple modelling, a good agreement is found with velocities derived from the optical proper motions measured in the ionized gas.

Subject headings: ISM: Herbig-Haro objects — ISM: individual (HH 1/2) — ISM: jets and outflows - ISM: molecules - stars: formation

\section{Introduction}

Bipolar outlows from embedded young stellar objects (YSOs) are perhaps one of the most spectacular manifestations of the star formation process. One of the best studied and brightest outflows is that of the Herbig-Haro (hereafter HH) 1/2 system (Reipurth 1993). The HH $1 / 2$ system lies in the Orion molecular cloud at $440 \mathrm{pc}$ and subtends a $\sim 2^{\prime}$ angle. The VLA 1 embedded source (Pravdo et al. 1985) drives a highly collimated jet that reaches atomic gas velocities of $\sim 480 \mathrm{~km} \mathrm{~s}^{-1}$ (Eislöffel et al. 1994) and produces shocks of $\sim 100 \mathrm{~km} \mathrm{~s}^{-1}$ at its main working surface or bow shock (Noriega-Crespo et al. 1989). At optical wavelengths there are at least 3 distinct jet flows arising within $5^{\prime \prime}$ of the VLA 1 source and the HH $1 / 2$ bow shocks display a complex morphology in high spatial resolution HST images (Bally et al. 2002). The optical jet is associated with a molecular outflow whose highvelocity (the "molecular jet") covers deprojected velocities of $15-80 \mathrm{~km} \mathrm{~s}^{-1}$ with respect to the ambient cloud (Moro-Martín et al. 1999). Because of these characteristics the HH 1/2 system is a perfect target to study the gas properties in a shock heated environment as well as the spatial distribution and the nature of these shocks. One of the promises of ISO was precisely to be able to discern between the different shocks, either C-type or J-type, occurring in a molecular environment.

\footnotetext{
${ }^{1}$ Based on observations with ISO, an ESA project with instruments funded by ESA Member States (especially the PI countries: France Germany, the Netherlands and the United Kingdom) and with the participation of ISAS and NASA
} 
We present here spectro-imaging observations of the VLA 1 counter-jet and the HH 2 region obtained between 5 and $17 \mu \mathrm{m}$ with the ISOCAM camera onboard ISO. We find that the $\mathrm{H}_{2}$ pure rotational lines $\mathrm{S}(2)$ to $\mathrm{S}(7)$ arise from two physically distinct regions : a faint extended warm gas component associated with the high-velocity CO jet, seen mainly in $\mathrm{S}(2)$, and several more compact peaks tracing hotter shocked gas in the individual knots A-L of $\mathrm{HH} 2$ detected in all lines. We report also on the presence of the [Ne III] $15.55 \mu \mathrm{m}$ and [Ne II] $12.8 \mu \mathrm{m}$ lines. HH 2 is the only outflow of low-luminosity where these infrared lines have been detected so far (see Cabrit et al. 1998).

\section{Observations}

All observations were obtained with the ISO satellite (Kessler et al., 1996) and the ISOCAM instrument (Cesarsky et al. 1996). The low resolution spectra $(\lambda / \Delta \lambda=40)$ between 5 and $17 \mu \mathrm{m}$ were obtained in revolution 691 with the Circular Variable Filter (CVF) with a pixel scale of $6^{\prime \prime}$ and a total field of view of $3^{\prime}$ centered on the HH 2 object. The last pipeline version of the data (OLP10) has been processed following the package developed at the Institut d'Astrophysique Spatiale, which removes reasonably well the problem of transients. The size (HPFW) of the Point Spread Function (PSF) is $\approx 6^{\prime \prime}$ for a pixel scale of $6^{\prime \prime}$.

In order to establish accurate astrometry, we used a second CVF map containing the optically visible Cohen-Schwartz (CS) star, taken in revolution 873 with a $3^{\prime \prime}$ pixel scale. This second dataset was processed in the same way as mentioned above. Unfortunately, internal reflections between the CVF and the field lens produced spurious ghosts of the CS star, which is a strong IR emitter, over the full field, preventing any quantitative analysis of the $\mathrm{H}_{2}$ line emission in the second data set. However, the presence of the VLA 1 protostar in both data cubes allows to derive an accurate astrometry for the first CVF image taken with a $6^{\prime \prime}$ pixel scale. The data are presented in Figure 1. Coordinates are offsets (arcsec) relative to the position of VLA1 : $\alpha_{2000}=05^{h} 36^{m} 22.6^{s}, \delta_{2000}=-06^{\circ} 46^{\prime} 25^{\prime \prime}$. The interstellar extinction towards HH 2 was estimated by Hartmann \& Raymond (1984), who measured typical reddenings $E(B-V)=0.11-0.44$. Based on the extinction curve of Rieke \& Lebofsky (1985), it appears that the flux dereddening corrections are negligible and we use uncorrected flux values in what follows. 


\section{Results}

Individual CVF spectra towards various optical knots in $\mathrm{HH} 2$ are displayed in the panels of Fig. 1b. Knot positions are referred by letters A to L following the nomenclature of Eisloeffel et al. (1994). At most positions, the bulk of the emission in the 5-17 $\mu \mathrm{m}$ range comes from the $\mathrm{H}_{2}$ pure rotational lines $\mathrm{S}(2)$ to $\mathrm{S}(7)$. An exception is the region near $\mathrm{HH} 2 \mathrm{H}$ (positions H,B,D) where the fine structure ionic lines [Ne II] $12.81 \mu \mathrm{m}$ and [Ne III] $15.55 \mu \mathrm{m}$ are also detected, and even dominate over $\mathrm{H}_{2}$ lines at the nominal position of knot $\mathrm{H}$. The spatial distribution of emission flux in the $\mathrm{H}_{2} \mathrm{v}=0-0 \mathrm{~S}(2), \mathrm{S}(3), \mathrm{S}(5)$ lines and in the [Ne II] $12.8 \mu \mathrm{m}$ ionic line is illustrated in Fig. 1a and Fig. 1c. Three types of morphologies are observed, depending on the line excitation :

(i) The ionic lines [Ne II] and [Ne III] show a single peak towards knot $\mathrm{H}$ (of typical size $\approx 7^{\prime \prime}$ at HPFW). (ii) The intermediate excitation lines $\mathrm{S}(3)$ and $\mathrm{S}(5)$ show two peaks of comparable brightness, one encompassing $\mathrm{E}$, and another peak centered between knots $\mathrm{H}$ and $\mathrm{D}$, shifted by $3^{\prime \prime}$ from the ionic line peak. (iii) The low energy $\mathrm{S}(2)$ line in Fig. 1a shows a single peak (of size 13") encompassing knots E and F. The emission peaks at the tip of the molecular jet 15" downstream the CO brightness peak. The lowest contours of $\mathrm{S}(2)$ emission reveal a broad pedestal that points towards VLA1/4 (Fig. 1a). Note that the first contour is at $5 \sigma$ above the map noise level. The pedestal overlaps well with the CO "jet" and suggests that both the $\mathrm{CO}$ and $\mathrm{H}_{2}$ emissions are somewhat related, although tracing different regions in the "jet".

The overall agreement between the brightness distributions of the pure $\mathrm{H}_{2}$ rotational lines and the higher excitation line 2.12 $\mu \mathrm{m}$ 1-0 S(1) (Davis et al. 1994) is good. Comparison of the line intensities indicates that the $\mathrm{H}_{2}$ pure rotational lines are collisionally excited (Wolfire \& Königl 1991). We show in the right panels of Figure 1 the $\mathrm{H}_{2}$ rotational diagrams obtained towards three representative positions : a region in the CO jet (polygon in Fig. 1a), knot $\mathrm{HH} 2 \mathrm{E}$ (peak of $\mathrm{H}_{2}$ no ionic line emission) and knot $\mathrm{HH} 2 \mathrm{D}$ (both ionic and molecular features). Overall, the fluxes of the lines $\mathrm{S}(\mathrm{J} \geq 3)$ match well a linear fit at all positions. The excitation temperature $\mathrm{T}_{\mathrm{ex}}$, the ortho to para ratio (OTP), and the total $\mathrm{H}_{2}$ column density of this "hot" component are estimated from a common linear fit to the temperature for the ortho and para species in the excitation diagram, and are summarized in Table 1.

Our analysis shows evidence for a hot gas layer with a temperature in the range 970$1400 \mathrm{~K}$, total $\mathrm{H}_{2}$ column density ranging between $0.18(\mathrm{H})$ and $1.6 \times 10^{19} \mathrm{~cm}^{-2}(\mathrm{E})$, and OTP ratio ranging between $1.2(\mathrm{E})$ and $2.5(\mathrm{H})$. There is no spatial trend in the values, i.e. upstream knots do not have a higher OTP ratio. Interestingly, there appears to be some correlation between variations in OTP and $\mathrm{T}_{\mathrm{ex}}$ : the smallest OTP (1.2) is found towards $\mathrm{HH} 2 \mathrm{E}$, which has among the lowest $\mathrm{T}_{\mathrm{ex}}$, while the highest OTP (2.5) is found towards HH 
$2 \mathrm{H}$, which has the highest $\mathrm{T}_{\mathrm{ex}}$. Knots $\mathrm{A}, \mathrm{C}, \mathrm{D}$, and $\mathrm{L}$ fall in between these two extremes. Knots B,K,G are the only positions that deviate from this trend (low $\mathrm{T}_{\mathrm{ex}}$ but OTP close to 2 ). The OTP ratios measured are systematically lower than the LTE value at all positions but $\mathrm{H}$. It is consistent with the low-excitation temperatures measured in the hot gas if $\mathrm{H}_{2}$ is excited by means of a C-shock (see e.g. Wilgenbus et al. 2000).

The rotational diagrams show that, at each position, the population of the upper level of the $\mathrm{S}(2)$ line lies well above the hot component fit to the other transitions. This implies that the pedestal $\mathrm{H}_{2}$ emission is dominated by a second gas component at a lower temperature, discussed in Sect. 4.3

\section{Discussion}

\subsection{Ionic emission in $\mathrm{HH} \mathbf{2 H}$}

The detection of the [Ne II] $12.8 \mu \mathrm{m}$ line towards $\mathrm{HH} 2 \mathrm{H}$ is a clear signature of J-shocks with velocities above $60 \mathrm{~km} \mathrm{~s}^{-1}$ (Hollenbach \& McKee 1989). The presence of [Ne III] is consistent also with the detection of the [Si II] $34.8 \mu \mathrm{m}$ line in $\mathrm{HH} 2$, which suggests shock velocities of $100-140 \mathrm{~km} \mathrm{~s}^{-1}$ (Molinari \& Noriega-Crespo 2002). That [Ne II] and [Ne III] are detected only towards $\mathrm{HH} 2 \mathrm{H}$ is in line with the particularly high excitation of this knot in the optical (Böhm \& Solf 1992), and its proper motion larger than $400 \mathrm{~km} \mathrm{~s}^{-1}$ (Bally et al. 2002). The high excitation of $\mathrm{HH} 2 \mathrm{H}$ is attributed to it being the current location of the jet working surface (Bally et al. 2002).

The shock speeds inferred in knot $\mathrm{H}$ exceed by far the speed at which molecules are dissociated $\left(\sim 70 \mathrm{~km} \mathrm{~s}^{-1}\right.$ for a C-shock at $\mathrm{n}_{0}(\mathrm{H})=10^{4} \mathrm{~cm}^{-3}$; LeBourlot et al. 2002, and $\sim 25 \mathrm{~km} \mathrm{~s}^{-1}$ for a J-shock; Hollenbach \& McKee 1989). This is consistent with the $\mathrm{H}_{2}$ peak in $\mathrm{S}(5)$ being spatially shifted from $\mathrm{HH} 2 \mathrm{H}$. A similar shift was seen in the $\mathrm{H}_{2}$ 1-0 $\mathrm{S}(1)$ line (Noriega-Crespo \& Garnavich, 1994). It indicates that the $\mathrm{H}_{2}$ comes from a separate, lower velocity shock not physically associated with knot $\mathrm{H}$.

\subsection{Shock-excitation of the hot $\mathrm{H}_{2}$ component in $\mathrm{HH}$ knots}

Models of non-dissociative J-shocks (Wilgenbus et al. 2000) or dissociative J-shocks with $\mathrm{H}_{2}$ reformation (Flower et al. 2003) fail to reproduce the CVF data as the predicted line intensities, expecially $\mathrm{S}(3)-\mathrm{S}(5)$, are much too weak, or the required shock velocity is too low $\left(\sim 10 \mathrm{~km} \mathrm{~s}^{-1}\right)$. On the contrary, everywhere in $\mathrm{HH} 2$, the recent models of planar 
C-shocks (Le Bourlot et al. 2002; Cabrit et al. 2003) provide a much better match with the observations.

Comparison with the observations favors models with velocity in the range $20-30 \mathrm{~km} \mathrm{~s}^{-1}$ and a preshock density in the range $10^{5}-10^{4} \mathrm{~cm}^{-3}$ respectively (see the rotational diagrams in Fig. 1). Observations of higher- $\mathrm{J} \mathrm{H}_{2}$ lines would allow to better constrain the density in the pre-shock gas. This range of densities also agrees with the determinations obtained in the ambient molecular cloud (Girart et al. 2002). The OTP ratio in the pre-shock gas is found $\simeq 1$. The observed variations in the OTP ratio from knot to knot can be produced by changes in the shock speed or preshock density, and do not require variations in the initial OTP ratio in the preshock gas. The characteristic size of the shock emitting region is constrained to be of the order of $0.8^{\prime \prime}-1.7^{\prime \prime}$ depending on the individual knots, which compares well with their size in the optical.

The above shock velocities are derived from planal models whereas HST images provide large evidence for numerous bow-shaped features in HH 2 (Bally et al. 2002). For several knots near the leading edge of HH 2 where the geometry is rather simple and bow-shaped features easily identified (e.g. F, E, L), the shock velocities compare well with the proper motions of the bow wings in the optical (e.g. $50-60 \mathrm{~km} \mathrm{~s}^{-1}$ at E) taking into account the obliquity of the shock with respect to the direction of the motion.

The absence of spatial trend in the OTP values probably results from the various shocks associated with the mini bows, unresolved in our CVF images. Each of these shocks modify the OTP ratio depending on its own excitation conditions.

\subsection{Warm extended $\mathrm{H}_{2}$ component}

An upper limit to the temperature of the extended warm component in the $\mathrm{H}_{2}$ pedestal can be derived from the ratio of the $\mathrm{S}(2)$ and $\mathrm{S}(3)$ lines (adopting an OTP ratio similar to that in the ambient cloud, close to 1 ). We find $\mathrm{T} \leq 420 \mathrm{~K}$ at the $\mathrm{H}_{2}$ peak and $\mathrm{T} \leq 380 \mathrm{~K}$ towards the $\mathrm{CO}$ jet. Note that a similar constraint, independent of the OTP ratio, is obtained by assuming that at most $50 \%$ of the $\mathrm{S}(4)$ flux comes from the pedestal.

Conversely, in the optically thin limit, we can also derive a lower limit to the temperature by imposing that the warm component contributes to most of the observed flux of the $\mathrm{J}=$ 2-1 line in the CO "jet" and assuming a standard abundance $[\mathrm{CO}] /\left[\mathrm{H}_{2}\right]=10^{-4}$ (Flower and Pineau des Forêts 1994 showed indeed that the CO abundance varies little in C-shocks). This temperature is found by equating expressions of the $\mathrm{H}_{2}$ column density derived from both tracers. Towards the region of maximum CO flux $\left(10-20 \mathrm{~K} \mathrm{~km} \mathrm{~s}^{-1}\right)$ in the jet the $\mathrm{H}_{2}$ 
$\mathrm{S}(2)$ line flux is $\simeq 8 \mathrm{mJy} /$ pixel, which yields a minimum temperature of $\simeq 240-300 \mathrm{~K}$ in the pedestal. This determination depends very little on the adopted OTP value. At the $\mathrm{H}_{2}$ peak the $\mathrm{CO}(2-1)$ emission is much weaker $\left(\simeq 6 \mathrm{~K} \mathrm{~km} \mathrm{~s}^{-1}\right)$ and we infer $\mathrm{T} \geq 400 \mathrm{~K}$.

The warm gas at $240-420 \mathrm{~K}$ detected in the pure $\mathrm{H}_{2}$ rotational lines of low-J is predominantly concentrated towards the tip of the high-velocity $\mathrm{CO}$ jet. The $\mathrm{S}(2)$ line suggests a warm gas column density of about $2 \times 10^{20} \mathrm{~cm}^{-2}$ at knots E-F and a corresponding mass of $\sim 2 \times 10^{-3} M_{\odot}$. The hot gas seen towards the high-velocity CO jet has a 10 times lower column density (Fig. 1). To reproduce the observed $\mathrm{J}=4$ population, as well as the limits on the excitation temperature, a low-velocity C-shock with a filling factor of 1 , implied by the extended character of the $\mathrm{S}(2)$ emission, is needed. Then, a rather slow shock at a velocity of $\sim 10-15 \mathrm{~km} \mathrm{~s}^{-1}$ into gas of density $\mathrm{n}(\mathrm{H}) \simeq 10^{5} \mathrm{~cm}^{-3}$, with an excitation temperature of $250 \mathrm{~K}$ would account for the $\mathrm{H}_{2}$ emission of the pedestal along the $\mathrm{CO}$ jet.

Observations of higher $\mathrm{J}$ CO rotational lines at higher angular resolution are needed to determine more precisely the physical conditions of the high-velocity CO gas and to compare it with that traced in $\mathrm{S}(2)$ and with molecular shock predictions.

J. Cernicharo acknowledges Spanish DGES for this research under grants PNAYA 20001784 and ESP2001-4516. ANC's research was carried out at the Jet Propulsion Laboratory, California Institute of Technology, under a contract with NASA; and partially supported by NASA-APD Grant NRA0001-ADP-096.

\section{REFERENCES}

Bally J., Heathcote, S., Reipurth, B., Morse, J., Hartigan, P., and Schwartz, R. 2002, AJ, 123,2667

Böhm, K.-H., Noriega-Crespo, A., \& Solf, J. 1992, ApJ, 416, 647

Böhm, K.-H., Solf, J., 1992, AJ, 104, 1193

Cernicharo, J., Reipurth, B., 1996, ApJ, 460, L57

Cabrit, S., Bontemps, S., Lagae, P.O., et al., 1998, in "The Universe as seen by ISO", pg 449, SP-427, eds P. Cox \& M.F. Kessler, ESA Publications Division, ESTEC, The Netherlands

Cabrit, S., 2003, in prep. 
Cesarsky, C.J., Abergel, A., Agnese, P., et al., 1996, A\&A, 315, L32

Davis, C.J., Eislöffel, J., Ray, T.P., 1994, ApJ, 426, L93

Eislöffel, J., Mundt, R., \& Böhm 1994, AJ, 108, 104

Flower, D.R., Pineau des Forets, G., 1994, MNRAS, 268, 724

Flower, D.R., LeBourlot, J., Pineau des Forets, G., Cabrit, S., 2003, MNRAS, in press

Girart, J.M., Viti, S., Williams, D.A., et al., 2002, A\&A, 388, 1004

Hartmann, L. \& Raymond, J.C., 1984, ApJ, 276, 560

Hollenbach, D., \& McKee, C. F. 1989, ApJ, 342, 306

Kessler, M.F., Steinz, J.A., Anderegg, M.E., et al., 1996, A\&A, 315, L27

Le Bourlot, J., Pineau des Forêts, G., Flower, D. R. \& Cabrit, S. 2002, MNRAS, 332, 985

Molinari, S., Noriega-Crespo, A., 2002, ApJ, 123, 2010

Moro-Martín, A., Cernicharo, J., Noriega-Crespo, A., Martín-Pintado, J., 1999, ApJ, 520, L111

Noriega-Crespo, A., Böhm, K.H., \& Raga, A.C. 1989, AJ, 98, 1388

Noriega-Crespo, A., Garnavich, P.M., 1994, AJ, 108, 1432

Reipurth, B., Heathcote, S., Roth, M.,Noriega-Crespo, A., Raga, A.C. 1993, ApJ, 408, L49

Rieke, G.H., Lebofsky, M.J., 1985, ApJ, 288, 618 ApJ, 481, 282

Wolfire, M.G., Konigl, A., 1991, ApJ, 383, 205

Wilgenbus,D., Cabrit, S., Pineau des Forets, G., Flower, D.R., 2000,A\&A, 356, 1010 
Table 1. Parameters of the hot molecular gas derived from rotational diagram analysis.

\begin{tabular}{cccc}
\hline \hline Knot & OTP & $\begin{array}{c}\mathrm{T}_{\mathrm{h}} \\
(\mathrm{K})\end{array}$ & $\begin{array}{c}\mathrm{N}_{\mathrm{h}}\left(\mathrm{H}_{2}\right) \\
\left(10^{18} \mathrm{~cm}^{-2}\right)\end{array}$ \\
\hline A & 1.7 & 1300 & 2.4 \\
$\mathrm{~B}$ & 1.9 & 1100 & 4.0 \\
$\mathrm{C}$ & 1.4 & 1070 & 2.8 \\
$\mathrm{D}$ & 1.5 & 1350 & 4.2 \\
$\mathrm{E}$ & 1.2 & 1030 & 16 \\
$\mathrm{G}$ & 2.1 & 1030 & 6.9 \\
$\mathrm{H}$ & 2.5 & 1440 & 1.8 \\
$\mathrm{~K}$ & 2.0 & 970 & 7.2 \\
$\mathrm{~L}$ & 1.4 & 1070 & 2.7 \\
\hline
\end{tabular}


Fig. 1 - (left) (a) : Intensity contour map of the $\mathrm{H}_{2}$ 0-0 $\mathrm{S}(2)$ line (pink) superposed on a [S II] color image of the $\mathrm{HH} 1 / 2$ region (Reipurth et al. 1993). Contour levels are 4, $710,15,20, \ldots$, $45 \mathrm{mJy} /$ pixel. We have superposed the emission of the high-velocity $\mathrm{CO}$ outflow in white contours. First contour and contour interval are 5 and $2.5 \mathrm{~K} \mathrm{~km} \mathrm{~s}^{-1}$ respectively. The position of the VLA 1-4 sources is indicated by yellow filled circles. Black dots mark the location of the knots A-L. (b1-b9) : CVF spectra at selected positions. Fluxes are in $\mathrm{mJy} /$ pixel. The jet spectrum is an average of the individual CV spectra between HH 2 and VLA 1 in the area of the polygon (yellow) on panel a. The wavelength of the pure rotational $\mathrm{H}_{2}$ transitions $\mathrm{S}(2)$ to $\mathrm{S}(7)$ is marked with red ticks. (c) : Superposed on the same [S II] picture : emission contour map of the lines $\mathrm{H}_{2} \mathrm{~S}(3)$ (c1), $\mathrm{H}_{2} \mathrm{~S}(5)$ (c2) and [Ne II] (c3). Contour levels are $0.1,0.2, \ldots 0.9$ times the brightness peak. The $\mathrm{H}_{2}$ intensity contour maps in panels (a) and (c) have been convolved with a gaussian of 9 " HPFW. (right) Rotational diagrams for the $\mathrm{H}_{2}$ rotational states $\mathrm{J}=3-9$. The logarithm of $\mathrm{N}_{\mathrm{J}} /\left(\mathrm{g}_{\mathrm{J}} \mathrm{g}_{\mathrm{s}}\right)$ is plotted against $\mathrm{E}_{\mathrm{J}} / \mathrm{k}_{\mathrm{b}}$, where $\mathrm{N}_{\mathrm{J}}$ is the beam-averaged column density, $\mathrm{g}_{\mathrm{J}}$ is the rotational degeneracy, $\mathrm{g}_{\mathrm{s}}$ is the spin degeneracy, and $\mathrm{E}_{\mathrm{J}}$ is the energy of the rotational level J. Open squares mark the data points. Blue straight lines show the best fit for a gas component of uniform temperature. The gas properties are given in each panel. The dashed lines in each panel correspond to the same temperature although they differ in the total column density for the orto and para species. Red dashed lines trace the fit to the warm extended component (pedestal). Green filled stars show the best planar C-shock model that accounts for the $J \geq 3$ data points. The parameters of the model (shock velocity $\mathrm{V}_{\mathrm{s}}$, hydrogen nuclei density $\mathrm{n}_{0}(\mathrm{H})$ and ortho to para ratio otp $\mathrm{p}_{0}$ in the pre-shock gas) and the pixel filling factor ff are given for $\mathrm{HH} 2 \mathrm{E}$ and $\mathrm{HH} 2 \mathrm{D}$. Below the panels of $\mathrm{HH} 2 \mathrm{E}$ and HH 2D are given the best fit parameters for planar C-shock models. 
Fig. 1.- 


\title{
Warm Molecular Hydrogen and Ionized Neon in the $\mathrm{HH} 2$ Outflow ${ }^{1}$
}

\author{
B. Lefloch ${ }^{1}$ \\ Laboratoire d'Astrophysique, Observatoire de Grenoble, BP 53, F-38041 Grenoble Cédex 9, \\ France \\ Bertrand.Lefloch@obs.ujf-grenoble.fr \\ J. Cernicharo ${ }^{2}$ \\ Consejo Superior de Investigaciones Cientificas, Instituto de Estructura de la Materia, \\ Serrano 123, E-28006 Madrid \\ S. Cabrit ${ }^{3}$ \\ LERMA, Observatoire de Paris, UMR 8112 du CNRS, France3 \\ A. Noriega-Crespo ${ }^{4}$ \\ SIRTF Science Center, Caltech JPL, Pasadena, CA 91125, USA \\ A. Moro-Martín ${ }^{5}$ \\ Steward Observatory, University of Arizona, Tucson, AZ, 85721, USA \\ D. Cesarsky ${ }^{6}$ \\ Max-Planck Institut für Extraterrestrische Physik, $85 \% 41$ Garching, Germany
}

\begin{abstract}
We report on spectro-imaging observations of the Herbig-Haro 2 outflow with the ISOCAM camera onboard the Infrared Space Observatory (ISO). The [Ne II] $12.81 \mu \mathrm{m}$ and [Ne III] $15.55 \mu \mathrm{m}$ lines are detected only towards the jet working surface ( $\mathrm{HH} 2 \mathrm{H}$ ), consistent with the high excitation of this knot in the optical range, while $\mathrm{H}_{2}$ pure rotational emission is found all over the shocked region HH 2. The low energy transition $\mathrm{S}(2)$ traces warm gas ( $\mathrm{T} \sim 400 \mathrm{~K}$ ) peaked towards knots $\mathrm{E}-\mathrm{F}$ and extended ejecta $(\mathrm{T} \sim 250-380 \mathrm{~K}$ ) with masses of a few $10^{-3} M_{\odot}$ in the high-velocity $\mathrm{CO}$ outflow extending between the powering source and HH 2. Such emission could arise from low-velocity C-type shocks
\end{abstract}


$\left(\mathrm{v} \simeq 10-15 \mathrm{~km} \mathrm{~s}^{-1}\right)$. The higher transitions $\mathrm{S}(3)-\mathrm{S}(7)$ trace the emission of hot shocked gas $(\mathrm{T}=1000-1400 \mathrm{~K}$ ) from individual optical knots in the HH 2 region. The ortho to para (OTP) ratio exhibits large spatial variations between $1.2(\mathrm{E})$ and $2.5(\mathrm{H})$, well below its value at LTE. The emission of the $\mathrm{S}(3)$ $\mathrm{S}(7)$ lines is well accounted for by planar C-shock models with a typical velocity $V_{\mathrm{s}}=20-30 \mathrm{~km} \mathrm{~s}^{-1}$ propagating into a medium of density $\mathrm{n}_{\mathrm{i}}=10^{4}-10^{5} \mathrm{~cm}^{-3}$ with an initial OTP ratio close to 1 in the pre-shock gas. In the leading edge of the jet, where the geometry of the emission allows a simple modelling, a good agreement is found with velocities derived from the optical proper motions measured in the ionized gas.

Subject headings: ISM: Herbig-Haro objects - ISM: individual (HH 1/2) — ISM: jets and outflows - ISM: molecules - stars: formation

\section{Introduction}

Bipolar outlows from embedded young stellar objects (YSOs) are perhaps one of the most spectacular manifestations of the star formation process. One of the best studied and brightest outflows is that of the Herbig-Haro (hereafter HH) $1 / 2$ system (Reipurth 1993). The HH $1 / 2$ system lies in the Orion molecular cloud at $440 \mathrm{pc}$ and subtends a $\sim 2^{\prime}$ angle. The VLA 1 embedded source (Pravdo et al. 1985) drives a highly collimated jet that reaches atomic gas velocities of $\sim 480 \mathrm{~km} \mathrm{~s}^{-1}$ (Eislöffel et al. 1994) and produces shocks of $\sim 100 \mathrm{~km} \mathrm{~s}^{-1}$ at its main working surface or bow shock (Noriega-Crespo et al. 1989). At optical wavelengths there are at least 3 distinct jet flows arising within $5^{\prime \prime}$ of the VLA 1 source and the HH 1/2 bow shocks display a complex morphology in high spatial resolution HST images (Bally et al. 2002). The optical jet is associated with a molecular outflow whose highvelocity (the "molecular jet") covers deprojected velocities of $15-80 \mathrm{~km} \mathrm{~s}^{-1}$ with respect to the ambient cloud (Moro-Martín et al. 1999). Because of these characteristics the HH $1 / 2$ system is a perfect target to study the gas properties in a shock heated environment as well as the spatial distribution and the nature of these shocks. One of the promises of ISO was precisely to be able to discern between the different shocks, either C-type or J-type, occurring in a molecular environment.

\footnotetext{
${ }^{1}$ Based on observations with ISO, an ESA project with instruments funded by ESA Member States (especially the PI countries: France Germany, the Netherlands and the United Kingdom) and with the participation of ISAS and NASA
} 
We present here spectro-imaging observations of the VLA 1 counter-jet and the HH 2 region obtained between 5 and $17 \mu \mathrm{m}$ with the ISOCAM camera onboard ISO. We find that the $\mathrm{H}_{2}$ pure rotational lines $\mathrm{S}(2)$ to $\mathrm{S}(7)$ arise from two physically distinct regions : a faint extended warm gas component associated with the high-velocity CO jet, seen mainly in $\mathrm{S}(2)$, and several more compact peaks tracing hotter shocked gas in the individual knots A-L of HH 2 detected in all lines. We report also on the presence of the [Ne III] $15.55 \mu \mathrm{m}$ and [Ne II] $12.8 \mu \mathrm{m}$ lines. HH 2 is the only outflow of low-luminosity where these infrared lines have been detected so far (see Cabrit et al. 1998).

\section{Observations}

All observations were obtained with the ISO satellite (Kessler et al., 1996) and the ISOCAM instrument (Cesarsky et al. 1996). The low resolution spectra $(\lambda / \Delta \lambda=40)$ between 5 and $17 \mu \mathrm{m}$ were obtained in revolution 691 with the Circular Variable Filter (CVF) with a pixel scale of $6^{\prime \prime}$ and a total field of view of $3^{\prime}$ centered on the HH 2 object. The last pipeline version of the data (OLP10) has been processed following the package developed at the Institut d'Astrophysique Spatiale, which removes reasonably well the problem of transients. The size (HPFW) of the Point Spread Function (PSF) is $\approx 6$ for a pixel scale of $6^{\prime \prime}$.

In order to establish accurate astrometry, we used a second CVF map containing the optically visible Cohen-Schwartz (CS) star, taken in revolution 873 with a $3^{\prime \prime}$ pixel scale. This second dataset was processed in the same way as mentioned above. Unfortunately, internal reflections between the CVF and the field lens produced spurious ghosts of the CS star, which is a strong IR emitter, over the full field, preventing any quantitative analysis of the $\mathrm{H}_{2}$ line emission in the second data set. However, the presence of the VLA 1 protostar in both data cubes allows to derive an accurate astrometry for the first CVF image taken with a $6^{\prime \prime}$ pixel scale. The data are presented in Figure 1. Coordinates are offsets (arcsec) relative to the position of VLA1 : $\alpha_{2000}=05^{h} 36^{m} 22.6^{s}, \delta_{2000}=-06^{\circ} 46^{\prime} 25^{\prime \prime}$. The interstellar extinction towards HH 2 was estimated by Hartmann \& Raymond (1984), who measured typical reddenings $E(B-V)=0.11-0.44$. Based on the extinction curve of Rieke \& Lebofsky (1985), it appears that the flux dereddening corrections are negligible and we use uncorrected flux values in what follows. 


\section{Results}

Individual CVF spectra towards various optical knots in HH 2 are displayed in the panels of Fig. 1b. Knot positions are referred by letters A to $\mathrm{L}$ following the nomenclature of Eisloeffel et al. (1994). At most positions, the bulk of the emission in the $5-17 \mu \mathrm{m}$ range comes from the $\mathrm{H}_{2}$ pure rotational lines $\mathrm{S}(2)$ to $\mathrm{S}(7)$. An exception is the region near HH $2 \mathrm{H}$ (positions H,B,D) where the fine structure ionic lines [Ne II] $12.81 \mu \mathrm{m}$ and [Ne III] $15.55 \mu \mathrm{m}$ are also detected, and even dominate over $\mathrm{H}_{2}$ lines at the nominal position of knot $\mathrm{H}$. The spatial distribution of emission flux in the $\mathrm{H}_{2} \mathrm{v}=0-0 \mathrm{~S}(2), \mathrm{S}(3), \mathrm{S}(5)$ lines and in the [Ne II] $12.8 \mu \mathrm{m}$ ionic line is illustrated in Fig. 1a and Fig. 1c. Three types of morphologies are observed, depending on the line excitation :

(i) The ionic lines [Ne II] and [Ne III] show a single peak towards knot H (of typical size $\approx 7^{\prime \prime}$ at HPFW). (ii) The intermediate excitation lines $\mathrm{S}(3)$ and $\mathrm{S}(5)$ show two peaks of comparable brightness, one encompassing E, and another peak centered between knots $\mathrm{H}$ and D, shifted by $3^{\prime \prime}$ from the ionic line peak. (iii) The low energy $\mathrm{S}(2)$ line in Fig. 1a shows a single peak (of size 13") encompassing knots $\mathrm{E}$ and $\mathrm{F}$. The emission peaks at the tip of the molecular jet $15^{\prime \prime}$ downstream the CO brightness peak. The lowest contours of S(2) emission reveal a broad pedestal that points towards VLA1/4 (Fig. 1a). Note that the first contour is at $5 \sigma$ above the map noise level. The pedestal overlaps well with the CO "jet" and suggests that both the $\mathrm{CO}$ and $\mathrm{H}_{2}$ emissions are somewhat related, although tracing different regions in the "jet".

The overall agreement between the brightness distributions of the pure $\mathrm{H}_{2}$ rotational lines and the higher excitation line $2.12 \mu \mathrm{m} 1-0 \mathrm{~S}(1)$ (Davis et al. 1994) is good. Comparison of the line intensities indicates that the $\mathrm{H}_{2}$ pure rotational lines are collisionally excited (Wolfire \& Königl 1991). We show in the right panels of Figure 1 the $\mathrm{H}_{2}$ rotational diagrams obtained towards three representative positions : a region in the $\mathrm{CO}$ jet (polygon in Fig. 1a), knot HH 2E (peak of $\mathrm{H}_{2}$ no ionic line emission) and knot HH 2D (both ionic and molecular features). Overall, the fluxes of the lines $\mathrm{S}(\mathrm{J} \geq 3)$ match well a linear fit at all positions. The excitation temperature $\mathrm{T}_{\mathrm{ex}}$, the ortho to para ratio (OTP), and the total $\mathrm{H}_{2}$ column density of this "hot" component are estimated from a common linear fit to the temperature for the ortho and para species in the excitation diagram, and are summarized in Table 1.

Our analysis shows evidence for a hot gas layer with a temperature in the range 970-

$1400 \mathrm{~K}$, total $\mathrm{H}_{2}$ column density ranging between $0.18(\mathrm{H})$ and $1.6 \times 10^{19} \mathrm{~cm}^{-2}(\mathrm{E})$, and OTP ratio ranging between $1.2(\mathrm{E})$ and $2.5(\mathrm{H})$. There is no spatial trend in the values, i.e. upstream knots do not have a higher OTP ratio. Interestingly, there appears to be some correlation between variations in OTP and $\mathrm{T}_{\mathrm{ex}}$ : the smallest OTP (1.2) is found towards HH $2 \mathrm{E}$, which has among the lowest $\mathrm{T}_{\mathrm{ex}}$, while the highest OTP (2.5) is found towards HH 
2H, which has the highest $\mathrm{T}_{\text {ex }}$. Knots $\mathrm{A}, \mathrm{C}, \mathrm{D}$, and $\mathrm{L}$ fall in between these two extremes. Knots B,K,G are the only positions that deviate from this trend (low $\mathrm{T}_{\mathrm{ex}}$ but OTP close to 2). The OTP ratios measured are systematically lower than the LTE value at all positions but $\mathrm{H}$. It is consistent with the low-excitation temperatures measured in the hot gas if $\mathrm{H}_{2}$ is excited by means of a C-shock (see e.g. Wilgenbus et al. 2000).

The rotational diagrams show that, at each position, the population of the upper level of the $\mathrm{S}(2)$ line lies well above the hot component fit to the other transitions. This implies that the pedestal $\mathrm{H}_{2}$ emission is dominated by a second gas component at a lower temperature, discussed in Sect. 4.3

\section{Discussion}

\subsection{Ionic emission in $\mathbf{H H ~} \mathbf{2 H}$}

The detection of the $[\mathrm{Ne}$ II $] 12.8 \mu \mathrm{m}$ line towards HH $2 \mathrm{H}$ is a clear signature of J-shocks with velocities above $60 \mathrm{~km} \mathrm{~s}^{-1}$ (Hollenbach \& McKee 1989). The presence of [Ne III] is consistent also with the detection of the [Si II] $34.8 \mu \mathrm{m}$ line in HH 2, which suggests shock velocities of $100-140 \mathrm{~km} \mathrm{~s}^{-1}$ (Molinari \& Noriega-Crespo 2002). That [Ne II] and [Ne III] are detected only towards HH $2 \mathrm{H}$ is in line with the particularly high excitation of this knot in the optical (Böhm \& Solf 1992), and its proper motion larger than $400 \mathrm{~km} \mathrm{~s}^{-1}$ (Bally et al. 2002). The high excitation of $\mathrm{HH} 2 \mathrm{H}$ is attributed to it being the current location of the jet working surface (Bally et al. 2002).

The shock speeds inferred in knot $H$ exceed by far the speed at which molecules are dissociated ( $\sim 70 \mathrm{~km} \mathrm{~s}^{-1}$ for a C-shock at $\mathrm{n}_{0}(\mathrm{H})=10^{4} \mathrm{~cm}^{-3}$; LeBourlot et al. 2002, and $\sim 25 \mathrm{~km} \mathrm{~s}^{-1}$ for a J-shock; Hollenbach \& McKee 1989). This is consistent with the $\mathrm{H}_{2}$ peak in $\mathrm{S}(5)$ being spatially shifted from HH 2H. A similar shift was seen in the $\mathrm{H}_{2}$ 1-0 $\mathrm{S}(1)$ line (Noriega-Crespo \& Garnavich, 1994). It indicates that the $\mathrm{H}_{2}$ comes from a separate, lower velocity shock not physically associated with knot $H$.

\subsection{Shock-excitation of the hot $\mathbf{H}_{2}$ component in $\mathbf{H H}$ knots}

Models of non-dissociative J-shocks (Wilgenbus et al. 2000) or dissociative J-shocks with $\mathrm{H}_{2}$ reformation (Flower et al. 2003) fail to reproduce the CVF data as the predicted line intensities, expecially $\mathrm{S}(3)-\mathrm{S}(5)$, are much too weak, or the required shock velocity is too low $\left(\sim 10 \mathrm{~km} \mathrm{~s}^{-1}\right)$. On the contrary, everywhere in $\mathrm{HH} 2$, the recent models of planar 
C-shocks (Le Bourlot et al. 2002; Cabrit et al. 2003) provide a much better match with the observations.

Comparison with the observations favors models with velocity in the range $20-30 \mathrm{~km} \mathrm{~s}^{-1}$ and a preshock density in the range $10^{5}-10^{4} \mathrm{~cm}^{-3}$ respectively (see the rotational diagrams in Fig. 1). Observations of higher-. $\mathrm{J} \mathrm{H}_{2}$ lines would allow to better constrain the density in the pre-shock gas. This range of densities also agrees with the determinations obtained in the ambient molecular cloud (Girart et al. 2002). The OTP ratio in the pre-shock gas is found $\simeq 1$. The observed variations in the OTP ratio from knot to knot can be produced by changes in the shock speed or preshock density, and do not require variations in the initial OTP ratio in the preshock gas. The characteristic size of the shock emitting region is constrained to be of the order of $0.8^{\prime \prime}-1.7^{\prime \prime}$ depending on the individual knots, which compares well with their size in the optical.

The above shock velocities are derived from planal models whereas HST images provide large evidence for numerous bow-shaped features in HH 2 (Bally et al. 2002). For several knots near the leading edge of HH 2 where the geometry is rather simple and bow-shaped features easily identified (e.g. F, E, L), the shock velocities compare well with the proper motions of the bow wings in the optical (e.g. $50-60 \mathrm{~km} \mathrm{~s}^{-1}$ at E) taking into account the obliquity of the shock with respect to the direction of the motion.

The absence of spatial trend in the OTP values probably results from the various shocks associated with the mini bows, unresolved in our CVF images. Each of these shocks modify the OTP ratio depending on its own excitation conditions.

\subsection{Warm extended $\mathbf{H}_{2}$ component}

An upper limit to the temperature of the extended warm component in the $\mathrm{H}_{2}$ pedestal can be derived from the ratio of the $\mathrm{S}(2)$ and $\mathrm{S}(3)$ lines (adopting an OTP ratio similar to that in the ambient cloud, close to 1 ). We find $\mathrm{T} \leq 420 \mathrm{~K}$ at the $\mathrm{H}_{2}$ peak and $\mathrm{T} \leq 380 \mathrm{~K}$ towards the CO jet. Note that a similar constraint, independent of the OTP ratio, is obtained by assuming that at most $50 \%$ of the $\mathrm{S}(4)$ flux comes from the pedestal.

Conversely, in the optically thin limit, we can also derive a lower limit to the temperature by imposing that the warm component contributes to most of the observed flux of the $\mathrm{J}=$ 2-1 line in the CO "jet" and assuming a standard abundance $[\mathrm{CO}] /\left[\mathrm{H}_{2}\right]=10^{-4}$ (Flower and Pineau des Forêts 1994 showed indeed that the CO abundance varies little in C-shocks). This temperature is found by equating expressions of the $\mathrm{H}_{2}$ column density derived from both tracers. Towards the region of maximum CO flux $\left(10-20 \mathrm{~K} \mathrm{~km} \mathrm{~s}^{-1}\right)$ in the jet the $\mathrm{H}_{2}$ 
$\mathrm{S}(2)$ line flux is $\simeq 8 \mathrm{mJy} /$ pixel, which yields a minimum temperature of $\simeq 240-300 \mathrm{~K}$ in the pedestal. This determination depends very little on the adopted OTP value. At the $\mathrm{H}_{2}$ peak the $\mathrm{CO}(2-1)$ emission is much weaker $\left(\simeq 6 \mathrm{~K} \mathrm{~km} \mathrm{~s}^{-1}\right)$ and we infer $\mathrm{T} \geq 400 \mathrm{~K}$.

The warm gas at $240-420 \mathrm{~K}$ detected in the pure $\mathrm{H}_{2}$ rotational lines of low- $\mathrm{J}$ is predominantly concentrated towards the tip of the high-velocity CO jet. The S(2) line suggests a warm gas column density of about $2 \times 10^{20} \mathrm{~cm}^{-2}$ at knots E-F and a corresponding mass of $\sim 2 \times 10^{-3} M_{\odot}$. The hot gas seen towards the high-velocity $\mathrm{CO}$ jet has a 10 times lower column density (Fig. 1 ). To reproduce the observed $\mathrm{J}=4$ population, as well as the limits on the excitation temperature, a low-velocity C-shock with a filling factor of 1 , implied by the extended character of the $\mathrm{S}(2)$ emission, is needed. Then, a rather slow shock at a velocity of $\sim 10-15 \mathrm{~km} \mathrm{~s}^{-1}$ into gas of density $\mathrm{n}(\mathrm{H}) \simeq 10^{5} \mathrm{~cm}^{-3}$, with an excitation temperature of $250 \mathrm{~K}$ would account for the $\mathrm{H}_{2}$ emission of the pedestal along the $\mathrm{CO}$ jet.

Observations of higher $\mathrm{J}$ CO rotational lines at higher angular resolution are needed to determine more precisely the physical conditions of the high-velocity $\mathrm{CO}$ gas and to compare it with that traced in $\mathrm{S}(2)$ and with molecular shock predictions.

J. Cernicharo acknowledges Spanish DGES for this research under grants PNAYA 20001784 and ESP2001-4516. ANC's research was carried out at the Jet Propulsion Laboratory, California Institute of Technology, under a contract with NASA; and partially supported by NASA-APD Grant NRA0001-ADP-096.

\section{REFERENCES}

Bally J., Heathcote, S., Reipurth, B., Morse, J., Hartigan, P., and Schwartz, R. 2002, AJ, 123,2667

Böhm, K.-H., Noriega-Crespo, A., \& Solf, J. 1992, ApJ, 416, 647

Böhm, K.-H., Solf, J., 1992, AJ, 104, 1193

Cernicharo, J., Reipurth, B., 1996, ApJ, 460, L57

Cabrit, S., Bontemps, S., Lagae, P.O., et al., 1998, in "The Universe as seen by ISO", pg 449, SP-427, eds P. Cox \& M.F. Kessler, ESA Publications Division, ESTEC, The Netherlands

Cabrit, S., 2003, in prep. 
Cesarsky, C.J., Abergel, A., Agnese, P., et al., 1996, A\&A, 315, L32

Davis, C.J., Eislöffel, J., Ray, T.P., 1994, ApJ, 426, L93

Eislöffel, J., Mundt, R., \& Böhm 1994, AJ, 108, 104

Flower, D.R., Pineau des Forets, G., 1994, MNRAS, 268, 724

Flower, D.R., LeBourlot, J., Pineau des Forets, G., Cabrit, S., 2003, MNRAS, in press

Girart, J.M., Viti, S., Williams, D.A., et al., 2002, A\&A, 388, 1004

Hartmann, L. \& Raymond, J.C., 1984, ApJ, 276, 560

Hollenbach, D., \& McKee, C. F. 1989, ApJ, 342, 306

Kessler, M.F., Steinz, J.A., Anderegg, M.E., et al., 1996, A\&A, 315, L27

Le Bourlot, J., Pineau des Forêts, G., Flower, D. R. \& Cabrit, S. 2002, MNRAS, 332, 985

Molinari, S., Noriega-Crespo, A., 2002, ApJ, 123, 2010

Moro-Martín, A., Cernicharo, J., Noriega-Crespo, A., Martín-Pintado, J., 1999, ApJ, 520, L111

Noriega-Crespo, A., Böhm, K.H., \& Raga, A.C. 1989, AJ, 98, 1388

Noriega-Crespo, A., Garnavich, P.M., 1994, A.J, 108, 1432

Reipurth, B., Heathcote, S., Roth, M.,Noriega-Crespo, A., Raga, A.C. 1993, ApJ, 408, L49

Rieke, G.H., Lebofsky, M.J., 1985, ApJ, 288, 618 ApJ, 481, 282

Wolfire, M.G., Konigl, A., 1991, ApJ, 383, 205

Wilgenbus,D., Cabrit, S., Pineau des Forets, G., Flower, D.R., 2000,A\&A, 356, 1010 
Table 1. Parameters of the hot molecular gas derived from rotational diagram analysis.

\begin{tabular}{cccc}
\hline \hline Knot & OTP & $\begin{array}{c}\mathrm{T}_{\mathrm{h}} \\
(\mathrm{K})\end{array}$ & $\begin{array}{c}\mathrm{N}_{\mathrm{h}}\left(\mathrm{H}_{2}\right) \\
\left(10^{18} \mathrm{~cm}^{-2}\right)\end{array}$ \\
\hline $\mathrm{A}$ & 1.7 & 1300 & 2.4 \\
$\mathrm{~B}$ & 1.9 & 1100 & 4.0 \\
$\mathrm{C}$ & 1.4 & 1070 & 2.8 \\
$\mathrm{D}$ & 1.5 & 1350 & 4.2 \\
$\mathrm{E}$ & 1.2 & 1030 & 16 \\
$\mathrm{G}$ & 2.1 & 1030 & 6.9 \\
$\mathrm{H}$ & 2.5 & 1440 & 1.8 \\
$\mathrm{~K}$ & 2.0 & 970 & 7.2 \\
$\mathrm{~L}$ & 1.4 & 1070 & 2.7 \\
\hline
\end{tabular}


Fig. 1 - (left) (a) : Intensity contour map of the $\mathrm{H}_{2}$ 0-0 $\mathrm{S}(2)$ line (pink) superposed on a [S II] color image of the $\mathrm{HH} 1 / 2$ region (Reipurth et al. 1993). Contour levels are 4, 7 10, 15, 20, .., $45 \mathrm{mJy} /$ pixel. We have superposed the emission of the high-velocity $\mathrm{CO}$ outflow in white contours. First contour and contour interval are 5 and $2.5 \mathrm{~K} \mathrm{~km} \mathrm{~s}^{-1}$ respectively. The position of the VLA 1-4 sources is indicated by yellow filled circles. Black dots mark the location of the knots A-L. (b1-b9) : CVF spectra at selected positions. Fluxes are in $\mathrm{mJy} /$ pixel. The jet spectrum is an average of the individual CV spectra between HH 2 and VLA 1 in the area of the polygon (yellow) on panel a. The wavelength of the pure rotational $\mathrm{H}_{2}$ transitions $\mathrm{S}(2)$ to $\mathrm{S}(7)$ is marked with red ticks. (c) : Superposed on the same [S II] picture : emission contour map of the lines $\mathrm{H}_{2} \mathrm{~S}(3)$ (c1), $\mathrm{H}_{2} \mathrm{~S}(5)$ (c2) and [Ne II] (c3). Contour levels are $0.1,0.2, \ldots 0.9$ times the brightness peak. The $\mathrm{H}_{2}$ intensity contour maps in panels (a) and (c) have been convolved with a gaussian of $9^{\prime \prime} \mathrm{HPFW}$. (right) Rotational diagrams for the $\mathrm{H}_{2}$ rotational states $\mathrm{J}=3-9$. The logarithm of $\mathrm{N}_{\mathrm{J}} /\left(\mathrm{g}_{\mathrm{J}} \mathrm{g}_{\mathrm{s}}\right.$ ) is plotted against $\mathrm{E}_{\mathrm{J}} / \mathrm{k}_{\mathrm{b}}$, where $\mathrm{N}_{\mathrm{J}}$ is the beam-averaged column density, $g_{\mathrm{J}}$ is the rotational degeneracy, $\mathrm{g}_{\mathrm{s}}$ is the spin degeneracy, and $\mathrm{E}_{\mathrm{J}}$ is the energy of the rotational level J. Open squares mark the data points. Blue straight lines show the best fit for a gas component of uniform temperature. The gas properties are given in each panel. The dashed lines in each panel correspond to the same temperature although they differ in the total column density for the orto and para species. Red dashed lines trace the fit to the warm extended component (pedestal). Green filled stars show the best planar C-shock model that accounts for the $J \geq 3$ data points. The parameters of the model (shock velocity $\mathrm{V}_{\mathrm{s}}$, hydrogen nuclei density $\mathrm{n}_{0}(\mathrm{H})$ and ortho to para ratio ot $\mathrm{p}_{0}$ in the pre-shock gas) and the pixel filling factor ff are given for $\mathrm{HH} 2 \mathrm{E}$ and $\mathrm{HH} 2 \mathrm{D}$. Below the panels of $\mathrm{HH} 2 \mathrm{E}$ and HH $2 \mathrm{D}$ are given the best fit parameters for planar C-shock models. 
Fig. 1.- 
This figure "fig1.jpg" is available in "jpg" format from: http://arxiv.org/ps/astro-ph/0305108v1 